\title{
Human Papilloma Virus
}

\section{Analysis of HPV FISH patterns in low and high grade Cervical Intraepithelial Neoplasia}

\author{
V.M.M. van Meegen \\ Dept of Molecular Biology- Maastricht University \\ v.vanmeegen@maastrichtuniversity.nl
}

\begin{abstract}
Human Papilloma Virus is the most common sexually transmitted infection, with an estimated $80 \%$ of sexually active men and women acquiring an infection at some point in their lifetime. $10-20 \%$ of infected individuals can not clear this infection effectively and consequentially are at risk for progression of Cervical Intraepithelial Neoplasia (CIN) to cancer. Presence of HPV can be determined using PCR and/or (Fluorescence) In Situ Hybridization. The aim of the performed experiments was to determine the general FISH patterns that are specifically linked to low grade and high grade CIN lesions and to investigate whether or not these patterns could be used to grade these lesions.

12 formalin fixed and paraffin embedded sections from one patient and 30 formalin fixed and paraffin embedded sections from different patients where used to perform a FISH procedure and to analyze the general FISH hybridization pattern for CIN 1,2 and 3. For the analysis 3 distinct patterns for the physical status of the virus were determined: episomal, integrated and mixed pattern. Also the presence of replication, load and the ratio between basal load and superficial load was analyzed to determine the general pattern.

Results show that load and physical status of the virus are not associated with the severity of the lesions. High loads are present in both high and low grade lesions. Also physical status of the virus is not different for the sections, episomal and mixed patterns are found in low and high grades. Only integrated pattern is a marker for severity, as this is only found in $\mathrm{CIN}_{3}$. Presence of replication is most common in $\mathrm{CIN}_{1}$, this might contribute to correct grading. The ratio between the load in the Basal layer and the load in the superficial layer

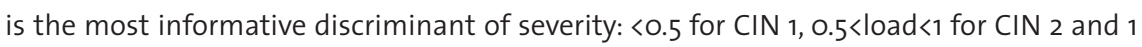
for $\mathrm{CIN} 3$. Based on these results it is possible to classify the severity of the lesion
\end{abstract}




\section{Keywords}

Human Papilloma Virus (HPV),Fluorescence in Situ hybridisation (FISH), Cervical Intraepithelial Neoplasia (CIN)

\section{Introduction}

Human Papilloma Virus (HPV) is the most common sexually transmitted disease in the world. An estimated $80 \%$ of all sexually active men and women will, at some point in their life, be infected with this virus ${ }^{[1]}$. As infection is, at first, without symptoms, it spreads easily and rates of infection appears to be increasing ${ }^{[2]}$

By now, over 200 types of HPV have been recognized, of which 58 genotypes are well investigated and characterized ${ }^{[2]}$. Typically two major classes of HPV are distinguished: high risk types and low risk types. The low risk types, amongst others HPV types 6,11,42,43 and 44 , are mainly associated to genital warts. However, the high risk types, e.g. types 16 , $18,31,33,45$ and 54 , are closely related to the development of several cancers ${ }^{[2]}$.

At present, HPV is associated mainly with (ano)genital cancer, but associations have been shown between HPV and tonsil cancer, larynx cancer and other head and neck cancers. Overall it is even estimated that HPV is causal in about $5 \%$ of all human cancers ${ }^{[1]}$. Although $80 \%$ of all sexually active people acquire an infection of HPV, the vast majority is able to clear the infection, without it causing problems for their health. In young women, an infection mostly clears within a 24 month time period ${ }^{[4]}$. An estimated $10-20 \%$ of the infected individuals however, is not able to clear the virus and the infection persists. These individuals are at risk for progression to precancers in the cervix - Cervical intra epithelial neoplasia (CIN). CIN lesions of a higher grade (CIN 2/3) are of high risk to progress into invasive cancer. The model theory of how HPV infects cervical epithelium and progresses to cancer is presented in figure 1.
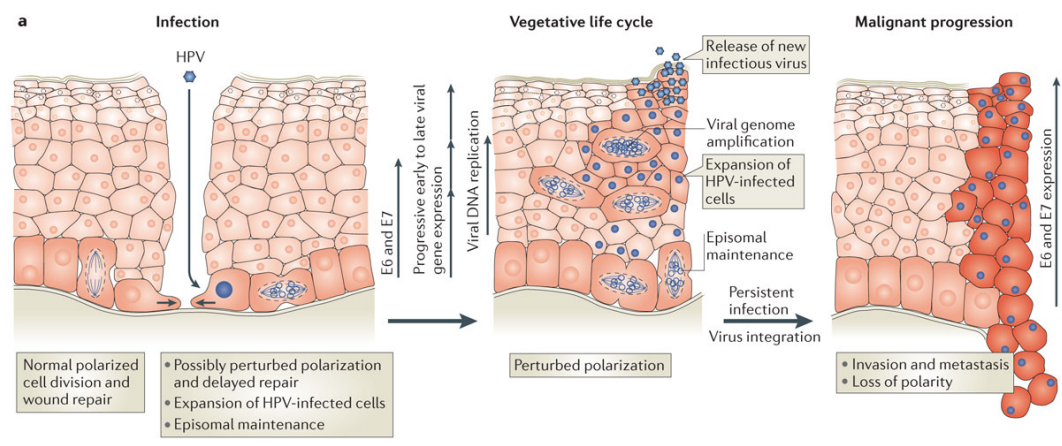

Figure 1. Mechanism of HPV infection and progression to malignancy

Model of how HPV infection might progress to an invasive tumour. The basal layer is infected through the skin. Viral gene products can be expressed and lead to new infection of cells via viral particles ${ }^{[5]}$. 


\section{Detection of HPV}

The primary method for detection of abnormalities in the cervix is the Papanicolao (Pap) smear. This method looks for changes in the transformation zone of the cervix. Then, according to the Bethesda System the findings can be recorded and scored according to CIN guidelines ${ }^{[6]}$. However, the Pap-smear method has its limitations and is not always accurate. Therefore, after a positive Pap-smear, other detection methods are used to confirm the presence of HPV. Usually a colposcopy is performed and, if no abnormal tissue is found at first sight, a biopsy is done. This biopsy can then be used for further analysis ${ }^{[3]}$.

As HPV cannot be grown in cell cultures and serological assays do not produce reliable results, detection of infection in this further analysis is usually done on a more molecular level ${ }^{[4]}$. For this reason PCR is now the most used technique to amplify the viral DNA signal and detect a HPV infection. PCR can also be used to accurately detect the specific types of HPV ${ }^{[7]}$ by verifying the presence of HPV DNA. However, the big drawback of using only amplification techniques is that only the presence of HPV can be established, but no information is gathered about the location or severity of this infection and the morphology of the sections analyzed.

To obtain this information a Fluorescence in Situ Hybridization (FISH) procedure could be used ${ }^{[8]}$. This technique can be used to visualize nucleic acid sequences by hybridizing a complementary probe ${ }^{[9]}$. Using labelled probes and an amplifying system as shown in figure 3 , the signal can be made visible when looked at under a fluorescence microscope. Using this technique, one cannot only visualize the HPV DNA, but also possible look the morphology of the sections.

When combining a PCR and the FISH technique, one could obtain not only specific information about the HPV genotypes that are present, but also about the localization of the infection and the physical status of HPV. However, contradictory to the pap-smear, nothing can be said about the severity of the abnormality, only about the presence of the HPV and, if FISH is used, about the physical status that the virus has in the sections.

The aim of the internship and the experiments performed was to see whether or not there is a specific FISH pattern for low and high grade CIN lesions and to determine what this pattern is exactly using the FISH technique.

If this pattern can be identified, the FISH technique could be used next to the Papsmear and other techniques to determine the CIN gradation of a lesion, which gives it an advantage over the now used PCR technique, which only shows presence of DNA, but cannot specify severity of a lesion. 


\section{Material and methods}

\section{Biological Materials}

To perform the analysis, two series of formalin fixed paraffin embedded tissue have been used. The first series where 12 sections of one patient from the department of Pathology at MUMC+. For this series no official CIN gradation was known, although there are scans with indications for a gradation, based on these marking the sections are considered CIN 3 lesions. The second series where 30 sections of 30 different patients from a series of 50 coupes, also from the department of Pathology. These coupes where CIN graded by a pathologist according to standard guidelines.

\section{FISH procedure}

To perform the FISH procedure for the FFPE's a standard protocol was used, as described by Hopman et al[1]1.

\section{Analysis}

After the staining procedures, slides were visually analyzed using a fluorescence microscope (Leica Metasystems). Four characteristics are determined at which analysis will be focused; physical status of the virus (Figure 2), the presence of Replication (Figure 3), Load and the ratio between the load at the basal layer and the load at the superficial side of the epithelium (Ratio basal layer:superficial).

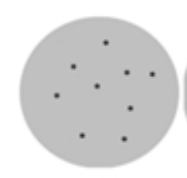

(A)

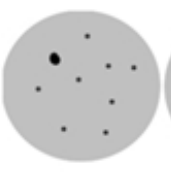

(B)

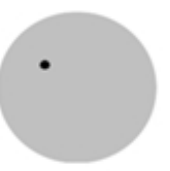

(C)

Figure 2. Depiction of the three typical FISH patterns that show the physical status of the virus s. A) Episomal pattern B) Mixed pattern, C) Integrated pattern ${ }^{[12,13,14]}$

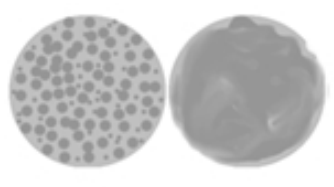

(A)
(B)

Figure 3. Depiction of replication A) Nucleus has multiple spots, all spots visible separately B) Nucleus has a multitude of signal, diffuse, spots are not visible separately. 


\section{Results}

\section{Comparison between low grade and high grade CIN lesions}

Typical examples of the patterns of infection found in the clinical samples are shown in figure 8. All the results for the analysis of these samples are shown in tables 1 and 2.

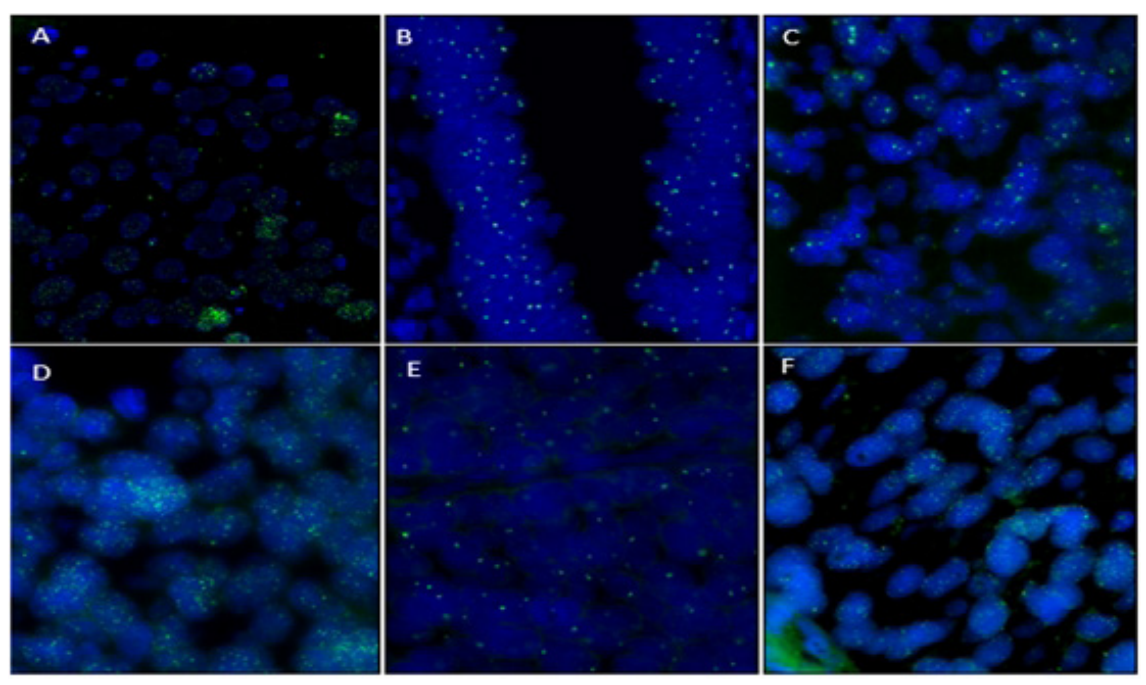

Figure 4. Depiction of the typical patterns as observed in the analysis of the clinical samples A+D: typical episomal pattern, $\mathrm{B}+\mathrm{E}$ : typical integrated pattern, $\mathrm{C}+\mathrm{F}$ typical mixed pattern.

Table 1. Results of the analysis of the CIN 1-3 series. Results are shown for Physical status of the virus, Presence of replication, Load and Ratio Basal Layer:Superficial. Results are split for $\mathrm{CIN}_{1}, \mathrm{CIN} 2$ and $\mathrm{CIN}_{3}$ graded sections.

\begin{tabular}{|c|c|c|c|c|c|c|c|}
\hline & Case & $\begin{array}{l}\text { HPB type } \\
\text { probe }\end{array}$ & $\begin{array}{l}\text { Physical status of } \\
\text { the virus }\end{array}$ & $\begin{array}{l}\text { Replication } \\
\text { Superficial layer }\end{array}$ & Load & $\begin{array}{l}\text { Ratio Basal layer : } \\
\text { Superficial layer }\end{array}$ & remarks \\
\hline \multirow{6}{*}{ CIN 1} & 21 & 16 & Episomal & & & & Positive area is too small to define pattern, very small coupe \\
\hline & 25 & 16 & Episomal & Not present & Intermediate & $<0.5$ & \\
\hline & 5 & 18 & Episomal & Present & High & $<0.5$ & Replication seem te be on the superficial epithelium \\
\hline & 7 & 31 & Episomal & Not present & High & $<0.5$ & \\
\hline & 2 & 31 & Episomal, Mixed & Present & Intermediate & $<0.5$ & \\
\hline & 3 & 31 & Episomal, Mixed & Present & High & $<0.5$ & \\
\hline \multirow{4}{*}{ CIN 2} & 13 & 16 & Episomal, Mixed & Not present & High & $0.5<\operatorname{load}<1$ & \\
\hline & 14 & 16 & Episomal & Not present & Intermediate & $0.5<$ load $<1$ & \\
\hline & 16 & 16 & Episomal & Not present & High & $0.5<1$ oad $<1$ & \\
\hline & 19 & 31 & Episomal & Present & High & $0.5<\operatorname{load}<1$ & Replication is only in a small area \\
\hline \multirow{7}{*}{ CIN 3} & 6 & 16 & Episomal, Mixed & Present & High & 1 & \\
\hline & 12 & 16 & Episomal & Present & High & 1 & Almost complete epithelium is positive \\
\hline & 17 & 16 & Integrated & Not present & Low & 1 & Only one spot per nucleus, in certain areas all nuclei positive \\
\hline & 23 & 16 & Episomal, Mixed & Not present & High & 1 & \\
\hline & 24 & 16 & Episomal & Not present & High & 1 & \\
\hline & 27 & 16 & Mixedl & Not present & High & 1 & \\
\hline & 8 & 31 & Episomal & Not present & High & 1 & \\
\hline
\end{tabular}


Table 2. Results of the analysis of the sections obtained from patient 31. Results are shown for Physical status of the virus, Presence of replication, Load and Ratio Basal layer: Superficial. Sections were all stained for HPV 16.

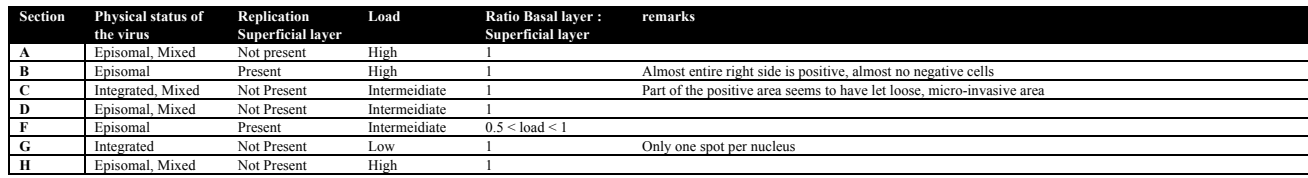

In tables 1 and 2 only the sections that were found positive are shown. For CIN 1 six out of nine analysed coupes where positive, for CIN 2 only four out of eight analyzed coupes are positive. For the CIN 3 coupes, in seven of ten coupes positivity was found. For the sections from patient 31, seven out of twelve coupes are positive, four coupes are negative and one coupe could not be analyzed because the tissue let loose of the slide during the FISH procedure.

\section{Physical status of the virus}

The most frequently observed FISH pattern is episomal, this in present in four of the CIN 1 graded tissues, three of the CIN 2 graded tissues and also three of the CIN 3 graded tissues. For the patient 31 sections, the episomal FISH pattern is observed in two of the cases. The second most observed FISH pattern is the episomal mixed, in total this is present in 8 of the cases. It is present twice in the CIN 1 cases, once in the CIN 2 cases and twice in the CIN 3 series. In the series from patient 31 this pattern is seen three times. A true mixed FISH pattern was only found once in a CIN 3 case. The integrated FISH pattern was found in two cases. Once in a CIN 3 graded case and once in the patient 31 sections. (suspected $\mathrm{CIN}_{3}$ ). In the patient 31 sections also an integrated, mixed FISH pattern was found once.

\section{Replication of HPV}

In total replication was observed in 8 cases. Three of these cases were CIN 1 graded, one was $\mathrm{CIN} 2$ graded. In CIN 3 graded cases replication was present in two of the cases. For patient 31(suspected $\mathrm{CIN}_{3}$ ) replication was also found in two of the sections. In 15 of the total cases replication was not present, in one of the $\mathrm{CIN} 1$ cases the presence of replication could not be analyzed. As shown in figure 5 for the $\mathrm{CIN} 1$ cases, the replication signal was more diffuse than for the $\mathrm{CIN} 3$ cases, in which all the spots could be seen separately. 


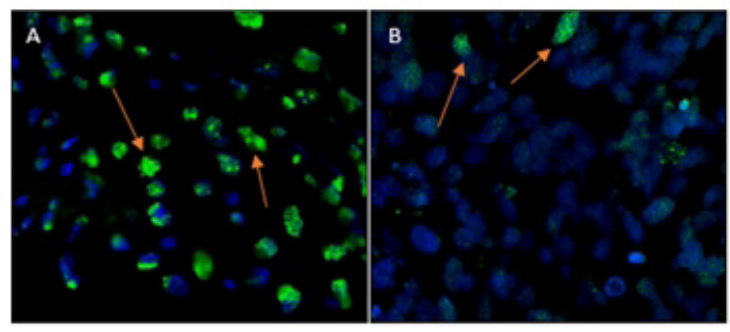

Figure 5. Representation of the two forms in which replication is observed. (A): CIN 1, replicative nuclei have a diffuse signal, spots are not visible separately. (B): CIN 3: spots in replicative nuclei are all distinguishable.

\section{Viral load:}

For the majority of the cases the load was high. This was found in three of the CIN 1 cases, three of the CIN 2 cases and 6 of the CIN 3 cases. For the sections from patient 31, high load was found in three of the cases. An intermediate load was found in two of the CIN1 cases and in 1 CIN 2 case. In the CIN 3 cases no intermediate load was observed whereas in patient 31 series (suspected CIN 3 cases) an intermediate load was found for three of the cases. Low load was only found twice in all the cases, both combined with an integrated pattern and both in $\mathrm{CIN}_{3}$ or $\mathrm{CIN}_{3}$ suspected cases.

\section{Ratio between the load in the Basal Layer compared to the Superficial load:}

For the Ratio Basal Layer: Superficial there was a clear distinction between the CIN 1, 2 and 3 grades cases. For all the CIN 1 cases, this ratio was below 0.5. For the CIN 2 graded cases, the ratio was below one, but higher than 0.5 whereas for CIN 3 graded cases the ratio was approximately 1.0 .

For the sections from patient 31, for all but one of the cases the ratio was approximately 1. For the other case (case F) this ratio was between 0.5 and 1.

Figure 6 is a representation of the ratio basal layer: superficial for a CIN 1 graded case and a CIN 3 graded case. 


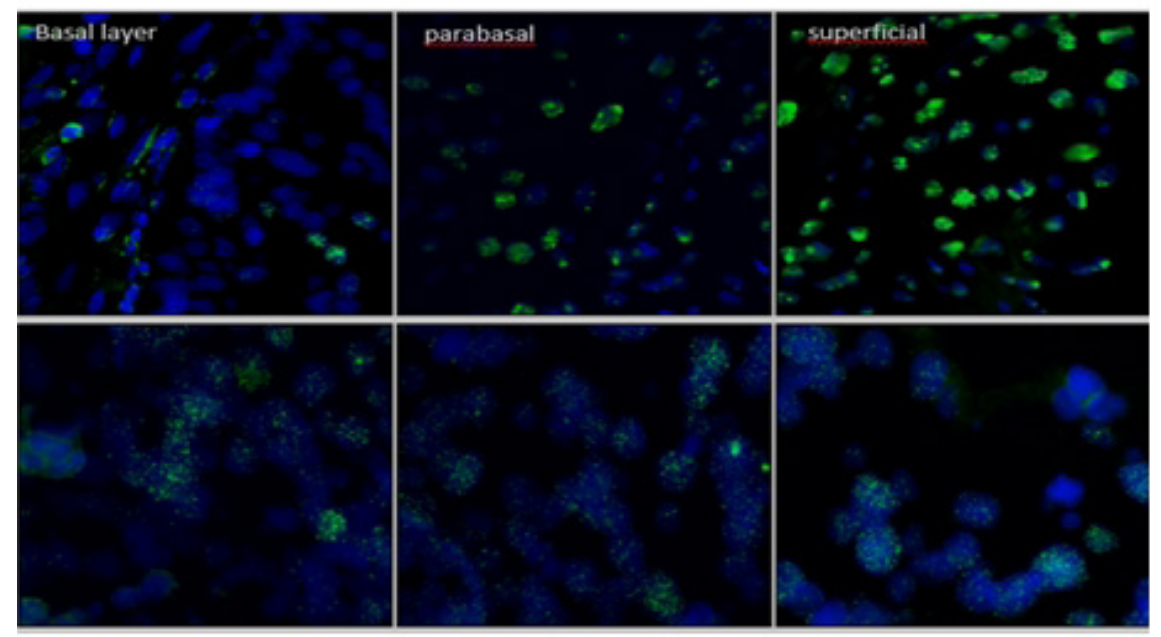

Figure 6. Upper panels: representation of a lesion area for CIN 1. Lower panels: representation of a lesion area for $\mathrm{CIN}_{3}$, both from basal, parabasal and superficial areas. Differences in the ratio between the basal layer load and superficial load are shown.

\section{Discussion/Conclusion}

\section{Comparison between low grade CIN and high grade CIN lesions}

For the sections of patient 31 only 7 of the slides were positive for HPV 16 . However, as the slides were not tested for HPV presence via other methods, it is not certain that the HPV 16 negative slides are not positive for other HPV types, as it is possible to have more than one HPV type in one lesion ${ }^{[1,16]}$.

In general, all of these slides had a ratio basal:superficial of 1, only one of the slides was deviant from this, with a higher load in the superficial epithelium than the basal layer. This could mean that this is a different CIN gradation. However, of this slide no gradation is given, so this theory can not be verified. Non of these slides are officially graded by a pathologist but, on scans of these coupes, areas with abberant tissue are marked with subsequent CIN marking of these areas. Based on these markings, slides A, B,C, D and G could be considered (suspected) $\mathrm{CIN}$ 3. Slide $C$ even contains a microinvasive area.

Of the other series, some of the coupes did not appear positive upon visual analysis. This could be due to fading of the signal, as the staining was performed some time ago. However as for a number of the other coupes signal intensity was still good, this is not likely. It is perhaps more probable that these lesions are either due to other types of HPV, which were not tested, or not even caused by HPV at all. 


\section{Comparison between all analyzed markers for gradation}

The most common pattern for all the coupes is the episomal or episomal mixed pattern. In all the cases, integrated FISH pattern was only observed twice. These integrated cases were labeled as $\mathrm{CIN}_{3}$ and suspected $\mathrm{CIN}_{3}$. As integration is associated with a higher severity this does not seem contradictory. This could also mean that when an integrated pattern is observed the section can always be classified as $\mathrm{CIN}_{3}$. The presence of episomal or mixed FISH patterns does not seem to be associated with the severity of the lesion as this presents itself in all the different gradations. Therefore physical status of the virus does not seem to be the most informative marker for the gradations, unless a high grade of integration is observed, which is associated with CIN 3.

Although in all grades of lesions replication was found, it seems to be more common in the $\mathrm{CIN} 1$ and 2 lesions. For the CIN 3 lesions it seems to not present as much (only two out of seven cases). What is remarkable is that for the CIN1 cases the signal in these nuclei seems more diffuse, the spots are not clearly distinctable, while in the CIN 3 the spots in these nuclei are all separately visible. This characteristic could contribute to determining the gradation of a lesion, however, replication alone does not appear to be a decisive marker for assigning a gradation.

When we compare load between all the lesions we can see that this is not a marker for the severity of the lesion and the gradation. Both in $\mathrm{CIN} 1$ and $\mathrm{CIN} 3$ cases with high loads are found. In fact, the only cases where a low load was found were the cases in which an integrated pattern was reported, being $\mathrm{CIN}_{3}$ cases. Thus, we could say, if load is low due to the integration of HPV in the host genome, it is most likely CIN3. However, a high or intermediate load does not seem to specifically linked to a gradation.

\section{Ratio between load in basal layer compared to the load in the superficial layer:}

This characteristic appears to be the most informative of the gradation. It is clear from the results that all the CIN 1 lesions have a much lower load near the basal layer than superficial. This is due to a much lower load around the basal layer than $\mathrm{CIN}_{3}$, where the load is evenly distributed throughout the epithelium. The ratio for $\mathrm{CIN}_{3}$ is approximately 1. For $\mathrm{CIN} 2$ the ratio is somewhere in between. It is not as low as $\mathrm{CIN} 1$, meaning the basal load is somewhat higher but it is also lower than for CIN3.The pattern in which the load is higher superficial than basal can be explained by the way in which HPV develops, from the basal layer, where it infects, to the maturation and production of viral particles higher in the epithelium ${ }^{[4,17]}$. 


\section{Problems}

The main problem with all of these experiments is that the number of coupes stained and analysed is relatively low. Therefore it is not possible to perform statistical analysis on the obtained results. Thus it is not known it all the previously mentioned and discussed results are significant.

\section{Conclusion}

We can conclude that the ratio basal layer:superficial is the most valuable discriminant for the gradation of the lesion. Also the presence of replicative pattern can be used as a discriminating factor. Load and physical status of the virus do not have a strong association with severity. Also the type of HPV does not seem to matter. This means that one can conclude that it is tissue from a CIN 1 lesion if the ratio basal layer:superficial is $<0.5$ and from a CIN 3 lesion if the ratio is approximately 1 . CIN 2 lesions show characteristics of both, showing replication and no replication evenly, and having a ratio between 0.5 and 1 . For a statistical analysis or predictive assay of these experiments there are a number of studies that could be conducted. One of the options would be to increase the total number of patients in the CIN 1, 2 and 3 series.

Testing the hypothesis should also be done by analyzing a "blinded" series, of which the severity is not known. If then, using the criteria as described in this paper, the grading is in accordance with the pathologist's opinion for a high percentage of cases, the predictive value of the determined FISH pattern is confirmed.

\section{Role of the student}

Valérie van Meegen was an undergraduate student in BioMedical Sciences working under the supervision of $\mathrm{AH}$ Hopman and $\mathrm{M}$ Ummelen when the research in this report was performed. The topic was proposed by the supervisor. The design of the experiment, the processing of the results as well formulation of the conclusions and the writing were done by the student.

\section{References}

1. Stanley M; HPV-immune response to infection and vaccination; Infectious agents and cancer;2010;5;10

2. Burd EM; Human Papillomavirus and Cervical Cancer; Clinical Microbiology Reviews; 2003;16;1;1-17

3. Muñoz N;Castellsagué X et al; Chapter 1: HPV in the etiology of human cancer; Vaccine; $2006 ; 24$

4. Molijn A; Kleter B et al; Molecular diagnosis of human papillomavirus (HPV) infections; journal of clinical virology;2005;S43-S51

5. Banks L; Pim D and Thomas M; Human tumour viruses and the deregulation of cell polarity in cancer; Nature Reviews Cancer, 2012;12;877-886 
6. Solomon D; Davey D; Kurman R et al; The 2001 Bethesa System: terminology for reporting results of cervical cytology; The Journal of the American Medical Association;2002;287;16;2114-2119

7. Villa LL; Denny L; Methods for detection of HPV infection and its clinical utility; International Journal of Gynecology and Obstetrics;2006;94;S71-S80

8. Birmer P; Bachtiary B et al; signal amplified colorimetric in situ hybridization for assessment of human papillomarvirus infection in cervical lesions;Modern Pathology 2001;14;7;702-709

9. Fluorescence in Situ Hybridization; Nature Methods;2005;2;237-238

10. Speel EJ; Schutte B et al; The effect of avidin-biotin interactions in detection systems for in situ hybridization; the journal of histochemistry and cytochemistry;1992;40;1;135-141

11. Hopman AHN; Remaekers FCS; Current protocols in cytometry; John Wiley and Sons,Inc; 1998;8.5.1 -8.5.22

12. Evans MF; Mount SL et al; biotinyl-tyrammide based in situ hybridization signal patterns distinguish human papillomavirus type and grade of cervical intraepithelial neoplasia;modern pathology;2002;15;12;1339-1347

13. Cooper K; Herrington CS et al; Episomal and integrated human papillomavirus in cervical neoplasia shown by non-isotopic in situ hybridization; journal of clinical pathology;1991;44;990-996

14. Kristiansen E, Jenkins A et al; Coexistence of episomal and integrated HPV16 DNA in squamous cell carcinoma of the cervix; journal of clinical pathology;1994;47;253-256

15. Chatuverdi AK et al; Human papillomavirus infections with multiple types; pattern of coinfection and risk of cervical disease; The Journal of Infectious Diseases;2001;2011;203;910-920

16. Rousseau MC; Pereira JS et al; Cervical coinfection with human papillomavirus (HPV) types as a predictor of acquisitions and persistence of HPV infection; Journal of Infectious Diseases;2001;15;1508-1517

17. Feller L; Khammissa RAG et al; epithelial maturation and molecular biology of oral HPV; Infectious agents and cancer;2009;4;16 